\title{
Nucleosome Depletion Alters the Chromatin Structure of Saccharomyces cerevisiae Centromeres
}

\author{
MICHAEL J. SAUNDERS, ${ }^{1}$ ELAINE YEH, ${ }^{1}$ MICHAEL GRUNSTEIN, ${ }^{2}$ AND KERRY BLOOM ${ }^{1 *}$ \\ Department of Biology, University of North Carolina, Chapel Hill, North Carolina 27599-3280 ${ }^{1}$ and Molecular Biology \\ Institute and the Department of Biology, University of California at Los Angeles, Los Angeles, California $90024^{2}$
}

Received 17 May 1990/Accepted 30 August 1990

\begin{abstract}
Saccharomyces cerevisiae centromeric DNA is packaged into a highly nuclease-resistant chromatin core of approximately 200 base pairs of DNA. The structure of the centromere in chromosome III is somewhat larger than a 160-base-pair nucleosomal core and encompasses the conserved centromere DNA elements (CDE I, II, and III). Extensive mutational analysis has revealed the sequence requirements for centromere function. Mutations affecting the segregation properties of centromeres also exhibit altered chromatin structures in vivo. Thus the structure, as delineated by nuclease digestion, correlated with functional centromeres. We have determined the contribution of histone proteins to this unique structural organization. Nucleosome depletion by repression of either histone $\mathrm{H} 2 \mathrm{~B}$ or $\mathrm{H} 4$ rendered the cell incapable of chromosome segregation. Histone repression resulted in increased nuclease sensitivity of centromere DNA, with up to $40 \%$ of CEN3 DNA molecules becoming accessible to nucleolytic attack. Nucleosome depletion also resulted in an alteration in the distribution of nuclease cutting sites in the DNA surrounding $C E N 3$. These data provide the first indication that authentic nucleosomal subunits flank the centromere and suggest that nucleosomes may be the central core of the centromere itself.
\end{abstract}

Accurate segregation of genetic material during both mitotic and meiotic cell division events depends on the centromere, a cis-acting DNA domain that provides a chromosomal attachment site for the mitotic apparatus. Centromere DNA sequences from the budding yeast Saccharomyces cerevisiae have been isolated and characterized $(4,6)$. The sequence requirements for centromere function have been defined through comparison of centromere DNAs $(6,13)$ and from in vitro alterations of conserved sequences followed by in vivo segregation assays. These studies revealed three centromere DNA elements (CDE I, II, and III) that are required for full mitotic and meiotic function $(6,13)$. The structural element of $S$. cerevisiae centromeres that is most conserved among the 11 centromeres sequenced to date is CDE III, a 25-base-pair (bp) DNA sequence that exhibits partial dyad symmetry (13). Single-point mutations within CDE III can entirely disrupt centromere function (20). CDE II (78 to 86 bp of $>90 \%$ AT DNA, with the A and T nucleotides commonly present in unbroken runs of 4 to 7 nucleotides) lies adjacent to CDE III. The third centromere DNA element, CDE I (PuTCACPuTG), resides distal to the CDE II. The sequence requirements for CDE I and II in centromere function are less stringent than those described above for CDE III. Centromeres with complete deletions of CDE I retain partial segregation function $(5,12)$. Similarly, single-base alterations in CDE II as well as small deletions or insertions (less than $50 \mathrm{bp}$ ) are tolerated to a great extent (8).

Molecular mapping techniques have been used to visualize the chromatin structure of $S$. cerevisiae centromeres (2). Approximately 220 bp of DNA encompassing the centromere DNA elements is folded into a highly nucleaseresistant conformation $(1,2)$. The single-point mutations in CDE III that inactivate centromere function eradicate the nuclease resistance associated with centromeric chromatin. The CEN3 CDE I and II mutations that retain partial CEN

\footnotetext{
* Corresponding author.
}

function exhibit chromatin conformations of altered dimensions (26). Mutations in which the length between CDE I and CDE III is increased or decreased exhibit lengthened or shortened protected structures, respectively. The chromatin particles associated with these defective centromeres can range from 145 to greater than $300 \mathrm{bp}$. Interestingly, mutants with less than 145 bp of centromere DNA exhibit protected structures that span 145 bp of chromatin DNA. Since this is the length of DNA protected by a nucleosome, the core structure must be composed of a subunit organization that at least correlates with the size of a nucleosome.

We have investigated the effects of nucleosome depletion on the centromere chromatin structure by using two $S$. cerevisiae strains in which the gene for either histone $\mathrm{H} 2 \mathrm{~B}$ or $\mathrm{H} 4$ can be repressed. Nucleosome loss results in increased sensitivity of centromere DNA and results in nucleosome depletion in the DNA surrounding CEN3. This result is consistent with the possibility that histone proteins are involved in determining the centromere structure in chromatin.

\section{MATERIALS AND METHODS}

Strains. S. cerevisiae $\mathrm{J} 17$ (MAT $\alpha$ his2 adel trpl met14 ura3-52) was used as the control strain. MHY102 [MATa htbl-1 htb2-2 ura3 his3-1 gallo can1-100 cyh2(pMH203, $\mathrm{pBM} 48)$ ] is the strain containing frameshift mutations of the two endogenous H2B genes, carrying a copy of the H2B gene under the control of the $G A L 10$ promoter on a centromere plasmid pMH203. UKY403 [MATa ade2-101 his3200 leu2-3 lys2-801 trpl ura3-52 thr tyr arg4-1 h4-1::LEU2 $h 4-2:: H I S 3$ (pUK421)] is the strain containing disruptions of the two endogenous histone $\mathrm{H} 4$ genes, carrying a copy of the H4-2 gene controlled by the $G A L I$ promoter on centromere plasmid pUK421.

Medium. J17 cells were grown in synthetic galactose (SG)-based medium $(0.67 \%$ yeast nitrogen base, $2 \%$ galactose, $0.5 \%$ Casamino Acids, $20 \mu \mathrm{g}$ each of adenine and uracil per $\mathrm{ml}$, and $50 \mu \mathrm{g}$ of tryptophan per $\mathrm{ml}$ ). MHY102 cells were 
grown in SG-His medium $(0.67 \%$ yeast nitrogen base, $2 \%$ galactose), and portions of the culture were switched to synthetic glucose (SD)-His medium $(0.67 \%$ yeast nitrogen base, $2 \%$ dextrose) and grown for $5 \mathrm{~h}$ until nucleosome depletion and $G_{2}$ cell cycle arrest occurred (9). UKY403 was grown selectively in SG-Trp medium $(0.67 \%$ yeast nitrogen base, $2 \%$ galactose, $0.5 \%$ Casamino Acids, $20 \mu \mathrm{g}$ each of adenine and uracil per $\mathrm{ml}$ ) and a portion of the cells was switched to prewarmed $\left(32^{\circ} \mathrm{C}\right) \mathrm{SD}$-based medium (same as SG-based medium, except $2 \%$ dextrose was used in place of the galactose) and incubated for $4 \mathrm{~h}$ until nucleosome depletion and $G_{2}$ cell cycle arrest occurred (18).

S-phase arrest by HU. UKY 403 cells were treated with hydroxyurea (HU) as previously described by Han et al. (9). Cultures were grown in SG-Trp medium to an $A_{660}$ of 0.2 (approximately $0.255 \times 10^{7}$ cells per $\mathrm{ml}$ ). HU was added to a final concentration of $0.3 \mathrm{M}$, and the culture was allowed to shake for $5 \mathrm{~h}$ at $32^{\circ} \mathrm{C}$. The cells were washed once in SD-Trp and suspended in SD-Trp containing 0.3 M HU and allowed to shake for $4 \mathrm{~h}$ at $32^{\circ} \mathrm{C}$. The cells were washed once in SG-Trp and suspended in SG-Trp in the absence of HU and allowed to grow for $14 \mathrm{~h}$ at $32^{\circ} \mathrm{C}$. At different times (see Fig. 4 and 5) samples were taken, the cells were washed once with water, and the pellets were stored at $4^{\circ} \mathrm{C}$ until preparation of nuclei.

$\mathbf{G}_{1}$ arrest of cells by $\alpha$ factor synchronization. $\alpha$ Factor $(10$ $\mu \mathrm{g} / \mathrm{ml}$ ) was used to arrest cells at $G_{1}$, as previously described by Kim et al. (18). UKY403 cells grown in SG-Trp medium to a density of approximately $0.38 \times 10^{7}$ cells per ml were treated with $\alpha$ factor and continued to shake at $32^{\circ} \mathrm{C}$ for $2 \mathrm{~h}$. Glucose was added to $2 \%$ and was further shaken at $32^{\circ} \mathrm{C}$ for $4 \mathrm{~h}$. After $2 \mathrm{~h}$ at $32^{\circ} \mathrm{C}$ the cells were treated again with $\alpha$ factor $(5 \mu \mathrm{g} / \mathrm{ml})$ to ensure $\mathrm{a} \mathrm{G}_{1}$ arrest. The cells were washed three times with SD-Trp to remove the $\alpha$ factor and were then grown in SD-Trp. Samples were taken at various times (see Fig. 5), and the cells were washed once with water and stored at $4^{\circ} \mathrm{C}$ until nuclei preparation.

Superhelical density of plasmid DNAs. Total yeast DNA was isolated as previously described by Bloom and Carbon (2). The DNA was electrophoresed on $1 \%$ agarose-chloroquine gels (27), transferred to nitrocellulose, and hybridized to either ${ }^{32} \mathrm{P}$-labeled pBR322 sequences to visualize the centromere plasmid or a 2.3-kilobase (kb) EcoRI fragment containing $2 \mu \mathrm{m}$ DNA to visualize the endogenous $2 \mu \mathrm{m}$ plasmid DNA.

Preparation and digestion of yeast nuclei. Yeast nuclei were prepared as described previously (2). MHY102 and UKY403 cells were grown in selective galactose-based media, and a portion of the cells were switched to prewarmed glucosebased media and incubated as described previously $(9,18)$ to repress transcription of the $\mathrm{H} 2 \mathrm{~B}$ gene (MHY102) or the $\mathrm{H} 4$ gene (UKY403) and induce nucleosome depletion and $G_{2}$ cell cycle arrest. Micrococcal nuclease digestion conditions of yeast nuclei were as previously described (2). For the DraI experiment (see Fig. 3) nuclei were suspended and washed in DraI digestion buffer $(10 \mathrm{mM}$ Tris hydrochloride [pH 7.0], $10 \mathrm{mM} \mathrm{MgCl} 2,20 \mathrm{mM} \mathrm{NaCl}, 0.1 \mathrm{mM}$ dithiothreitol, $1 \mathrm{mM}$ phenylmethylsulfonyl fluoride) and then incubated with three different concentrations of $\operatorname{DraI}(50,150$, and 300 $\mathrm{U} / \mathrm{ml}$ of nuclei) at $37^{\circ} \mathrm{C}$ for $30 \mathrm{~min}$. DraI digestion of yeast chromatin was terminated by the addition of EDTA to 50 mM. Chromatin samples were deproteinized, and the DNA was precipitated with ethanol. DNA samples were cleaved to completion with the appropriate restriction enzyme (see figure legends), and the samples were analyzed by electrophoresis on agarose slab gels containing $0.09 \mathrm{M}$ Tris borate
(pH 8.3) and 2.5 mM EDTA. Nucleic acids were transferred to nitrocellulose filters and hybridized to the 630-bp CEN3radiolabeled DNA fragment originating from the BamHI site and extending over the centromeric region of chromosome III (Fig. 1 and 2A) or the 900-bp HindIII-BamHI fragment flanking CEN3 (Fig. 2B, 3, and 4). DNA fragments were scanned on a Molecular Dynamics computing densitometer.

\section{RESULTS}

Two yeast strains have been constructed in which the sole functional gene for histone $\mathrm{H} 2 \mathrm{~B}$ (strain MHY102) or $\mathrm{H} 4$ (UKY403) has been placed under the control of the GAL10 or GAL1 promoter, respectively $(9,10,18)$. The viability of these strains requires growth in galactose-containing medium. Switching the cells to glucose-containing medium results in transcriptional repression of the $\mathrm{H} 2 \mathrm{~B}$ or $\mathrm{H} 4$ gene. New H2B or H4 synthesis is blocked and results in general nucleosome depletion on replication. The switched cells can complete only one round of DNA replication before synchronous arrest in the $G_{2}$ phase of the cell cycle $(9,18)$.

An indirect end-labeling mapping strategy was used to determine the chromatin structure of CEN3 and flanking sequences in MHY102 (H2B) or UKY403 (H4) in both galactose-grown cells and cells switched to glucose. Nuclei were prepared from wild-type control strain J17, MHY102 grown in galactose, and MHY102 switched to glucose (Fig. 1). Nuclei were partially digested with micrococcal nuclease. DNA was deproteinized and then digested with BamHI. CEN3 chromatin DNA was protected from micrococcal nuclease digestion in galactose-grown cells (Fig. 1, 360 to $627 \mathrm{bp}$ interval in lanes J17-GAL) with an extensive series of cutting sites seen at 160 -bp intervals in the region flanking CEN3. A number of faint cutting sites, 627 to $1,900 \mathrm{bp}$ interval, not seen in the wild-type chromatin lanes were apparent in the flanking regions (Fig. 1, lanes MHY102GAL). These additional cutting sites may result from a small proportion of cells (less than 5\%) (data not shown) in the population that have lost the plasmid pMH203 carrying the histone $\mathrm{H} 2 \mathrm{~B}$ gene. This would lead to nucleosomal depletion and cell cycle arrest in this subset of galactose-grown cells. Alternatively, the overproduction of $\mathrm{H} 2 \mathrm{~B}$ in galactose may perturb the stoichiometry among the four core histones that is required for faithful chromosome segregation (22).

Following a growth regime that elicits nucleosome depletion (Fig. 1, lanes MHY102-GLU [chromatin]), cleavage sites within the centromere core were apparent in the interval between 360 and 627 bp (Fig. 1, lanes MHY102GLU [10 to $45 \mathrm{~min}$ ]). These cutting sites mapped to CDE II and were identical to cutting sites seen in this region of deproteinized DNA control lanes (Fig. 1, lane N). The intensity of these cutting sites implies that only a portion of the DNA molecules in the population was exposed. In addition, cutting sites were seen in the flanking DNA that were not seen in the wild-type control (Fig. 1, lanes J17GAL). The distribution of bands in lanes MHY102-GLU resembled a composite of those seen in the lanes MHY102GAL (wild-type chromatin) and the naked DNA lanes (Fig. 1). These data are consistent with an altered distribution of chromatin proteins on a sizable fraction of the CEN 3 molecules in the population.

Figure 2 shows the results of micrococcal nuclease mapping studies in the strain UKY403 in which histone H4 could be transcriptionally regulated. The nuclease cutting pattern exhibited in the UKY403-GAL chromatin lanes (Fig. 2A and B) revealed the characteristic centromere core particle (Fig. 


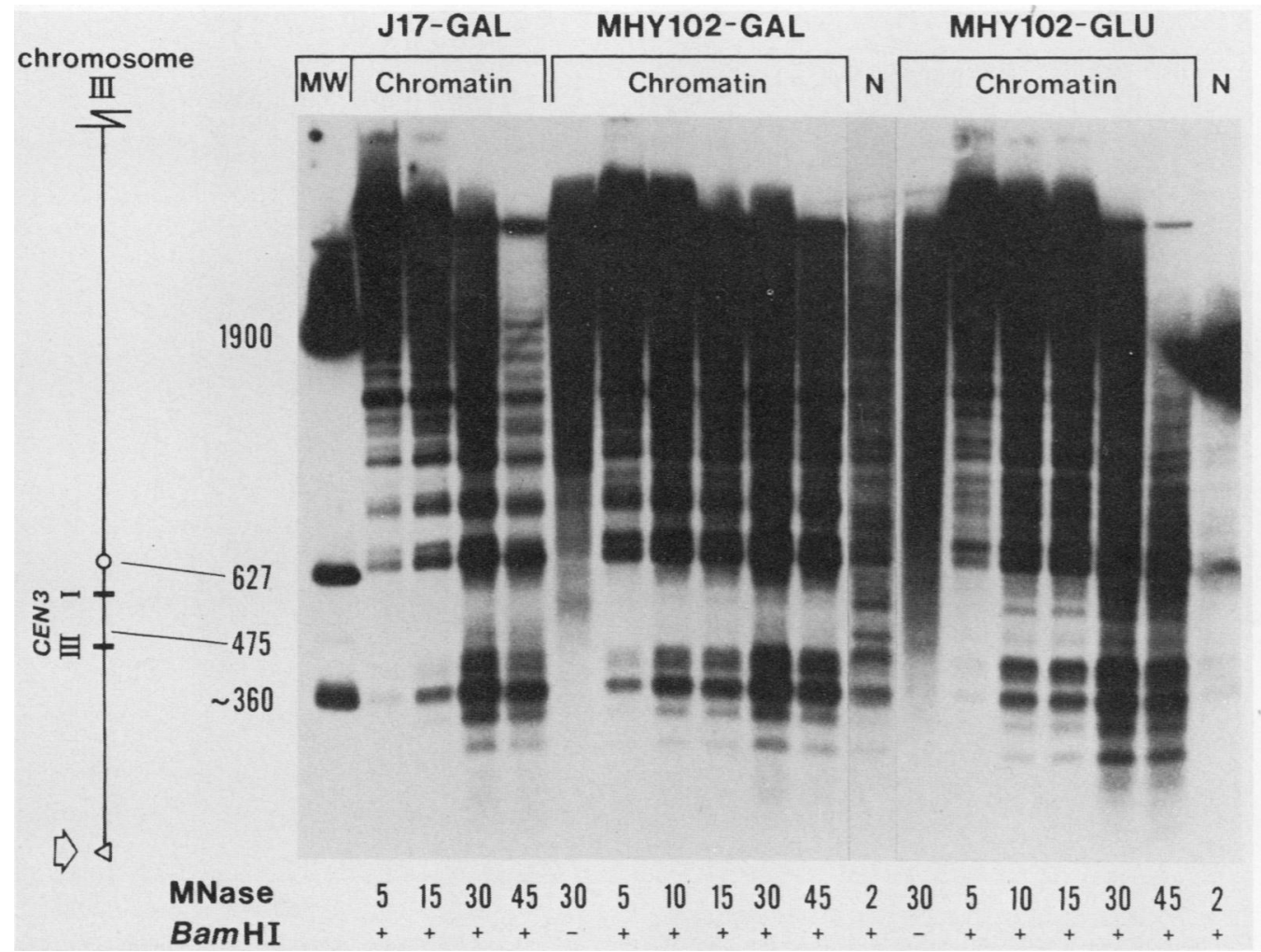

FIG. 1. Effects of histone H2B depletion on CEN3 chromatin structure. Strain MHY102 contains frameshift mutations of the endogenous copies of the histone H2B genes and the regulated copy of the histone H2B gene on the plasmid pMH203. Nuclei were prepared from J17 (lanes J17-GAL) and MHY102 cells grown under conditions of histone H2B induction (lanes MHY102-GAL) and repression (lanes MHY102-GLU). Nuclei were treated with 100 to $300 \mathrm{U}$ of micrococcal nuclease per $\mathrm{ml}$ for the number of minutes indicated below each lane. DNA in the naked control lanes (N) was deproteinized prior to micrococcal nuclease treatment. DNA samples were restricted with BamHI $(+)$ or left unrestricted $(-)$ and analyzed as described in Materials and Methods. To the left of the figure is a partial restriction map of the centromere region of chromosome III (map shows Bam HI, $\triangle$, Sau3AI, 0). Molecular size markers (lane MW) are 1,900-, 627-, 475-, and 360-bp fragments containing CEN3 DNA. The position of the markers relative to the partial restriction maps are indicated by lines.

2A, 360-630 bp; Fig. 2B, vertical bar) that was flanked by cutting sites at 160 -bp intervals. Nucleosome depletion resulted in increased sensitivity of $C E N 3$ DNA to micrococcal nuclease (Fig. 2A and 2B, lanes UKY403-GLU). Cutting sites located in CDE II were apparent and corresponded to cleavage sites in deproteinized CEN3 DNA (Fig. 2A). The nuclease-sensitive sites delineating the centromere core were less distinctive following nucleosome depletion. The general cleavage pattern in the UKY403-GLU lanes was intermediate between the patterns seen for wild-type chromatin and naked DNA (Fig. 2).

The structural integrity of the centromere protein complex was compromised on nucleosome depletion. The increase in nuclease accessibility of centromere DNA could be quantitated by measuring the appearance of an internal centromere fragment following digestion of chromatin DNA with restriction enzymes. We have taken advantage of naturally occurring recognition sites within CDE II (TTTAAA) for DraI. DraI recognizes three closely spaced sites in CDE II and has been used to distinguish chromatin structures in wild-type and mutated centromeres (26). Nuclei prepared from strain UKY403 grown in galactose or switched to glucose were incubated with DraI, as described in the legend to Fig. 3. Following digestion, the DNA samples were deproteinized, digested with HindIII, electrophoresed on a $1.4 \%$ agarose gel, and transferred to a nitrocellulose filter. A $0.9-\mathrm{kb}$ HindIII-BamHI fragment flanking CEN3 was used as a radiolabeled probe to visualize the intact HindIII fragment
(Fig. 3, $7.0 \mathrm{~kb}$, uppermost band), and the DraI-HindIII fragment $(1.38 \mathrm{~kb})$, representing digestion of the DraI sites in CDE II. The autoradiograph in Fig. 3 demonstrates that the centromeric DraI sites were more sensitive to digestion in glucose-grown cells than in galactose-grown cells, as visualized by an increase in intensity of the 1.38-kb HindIII-DraI fragment. The intensities of the 1.38-kb DraI digestion product and the 7.0-kb intact HindIII fragment were scanned with a laser densitometer to quantitate the area under the respective peaks. Scanning from a number of experiments revealed that at least one of the three DraI sites located in $C E N 3$ was cleaved in approximately $40 \%$ of the CEN3 DNA molecules following incubation with DraI (Fig. 3, lane GLU, $300 \mathrm{U} / \mathrm{ml}$ of nuclei), compared with less than $5 \%$ in galactose-grown cells $(300 \mathrm{U} / \mathrm{ml}$ of nuclei).

The accessibility of approximately half the centromeres to nucleolytic attack may reflect the portion of molecules that become exposed while replication ensues in the absence of histone synthesis. To examine whether centromere accessibility was correlated with progression through $\mathrm{S}$ phase, UKY 403 cells were first treated with $\mathrm{HU}$ in the presence of galactose under conditions that arrest DNA replication and cell division (9). After $5 \mathrm{~h}$ in HU plus galactose, 85 to $90 \%$ of the cells contained a single, large bud. Histone synthesis was repressed by switching cells to glucose while the HU treatment was continued. Nuclei were isolated from samples of cells at various points throughout this regime, and the chromatin DNA was subjected to DraI digestion as de- 


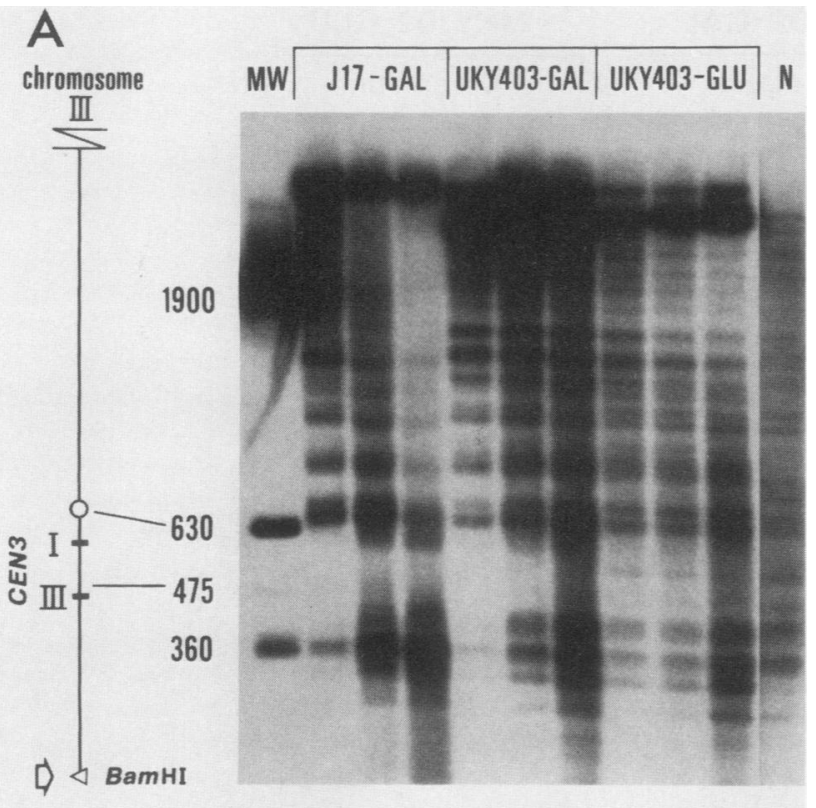

MNase $\begin{array}{lllllllll}5 & 15 & 30 & 5 & 15 & 30 & 5 & 15 & 30\end{array}$

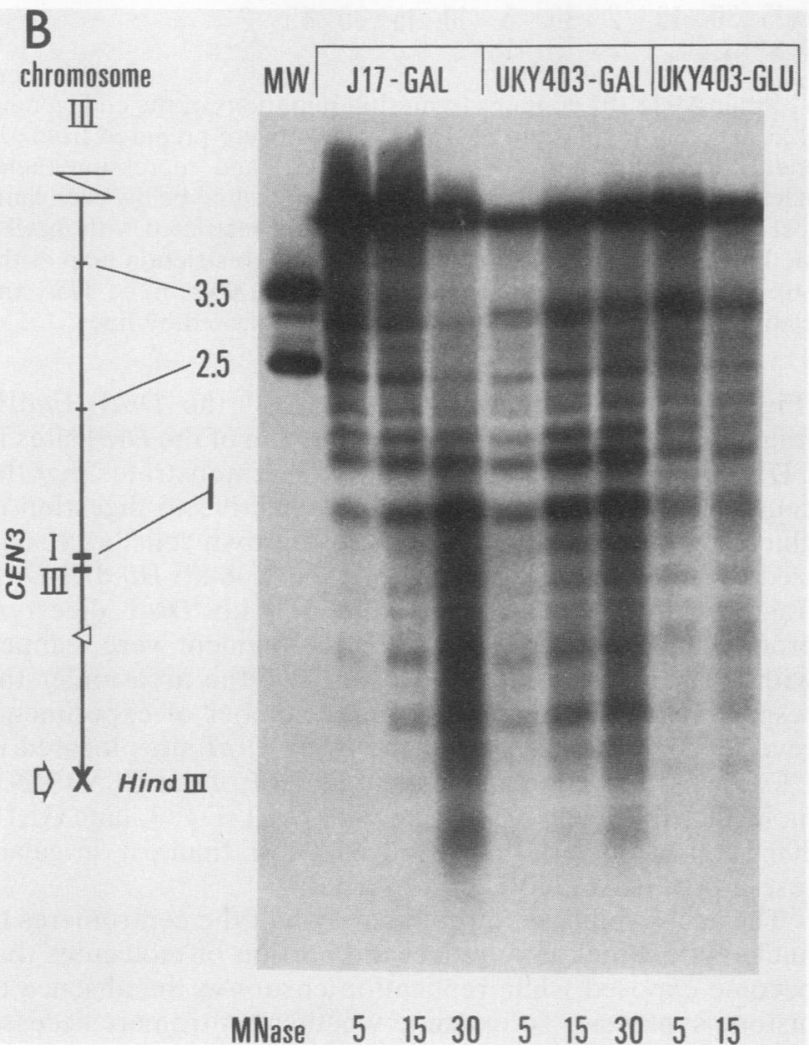

FIG. 2. Effects of histone $\mathrm{H} 4$ depletion on $C E N 3$ chromatin structure. Strain UKY403 contains disruptions of the two endogenous histone $\mathrm{H} 4$ genes and the regulated copy of the $\mathrm{H} 4$ gene on plasmid pUK421. Nuclei were prepared from J17 (lanes J17-GAL) and UKY403 cells under conditions of histone $\mathrm{H} 4$ induction (lanes UKY403-GAL) and repression (lanes UKY403-GLU). Nuclei were treated with micrococcal nuclease, as described in the legend to Fig. 1. A naked control lane $(N)$ was also prepared as described in the legend to in Fig. 1. (A) The BamHI site closest to CEN3 was used as

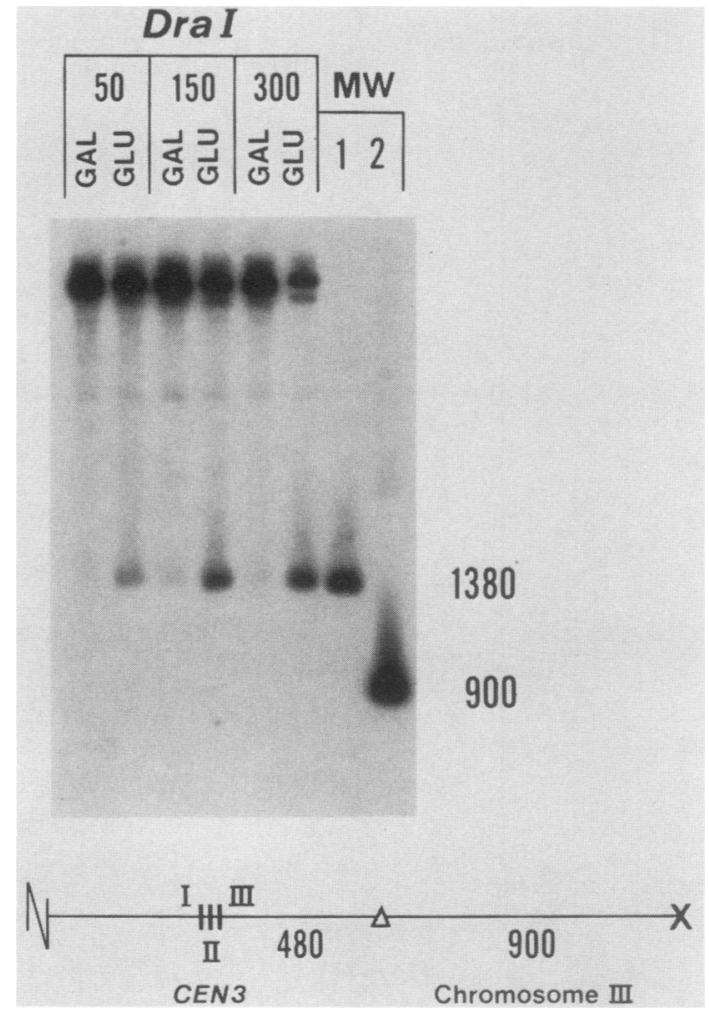

FIG. 3. Increase in DraI accessibility of $C E N 3$ chromatin occurring on nucleosome depletion. Nuclei were prepared from yeast strain UKY 403 grown in galactose medium (lanes GAL) or switched to glucose medium (lanes GLU) and digested with DraI as described in Materials and Methods. Galactose- and glucose-grown chromatin samples were paired according to DraI concentration. The $0.9-\mathrm{kb}$ HindIII-BamHI fragment flanking CEN3 was used as a radiolabeled probe to visualize the intact HindIII fragment $(7.0 \mathrm{~kb}$, uppermost band in autoradiograph) as well as digestion at the DraI sites located in CDE II by the appearance of a $1.38-\mathrm{kb}$ band (see the partial restriction map of the centromere region of chromosome III, which shows BamHI, $\triangle$, HindIII, $\mathrm{X}$, and DraI, |). Molecular size markers (lanes MW) were prepared by digestion of yeast genomic DNA with HindIII and DraI (MW lane 1, $1380 \mathrm{bp}$ ) or HindIII and BamHI (MW lane 2, 900 bp).

scribed above. Quantitation of the degree of digestion was performed by laser densitometry of autoradiographs (Fig. 4) to determine the percent of the 1.38-kb DraI digestion product relative to the intact 7.0-kb HindIII fragment. Only a portion of the centromeres (less than $10 \%$ ) were accessible to DraI cutting after $4 \mathrm{~h}$ in glucose in the absence of continued DNA replication (Fig. 4). When cells were shifted to glucose in the absence of $\mathrm{HU}, \mathrm{DraI}$ accessibility pro-

the end point for mapping. This strategy revealed the chromatin structure of both the chromosomal and the plasmid CEN3 DNA. (B) The HindIII site $1.3 \mathrm{~kb}$ from CDE III of CEN3 was used as the mapping reference point, in a strategy revealing the chromatin structure of only the chromosomal CEN3. The position of the protected centromere core is indicated by the vertical bar. Partial restriction maps of the centromere regions of chromosome III are included (map shows HindIII, X, BamHI, $\triangle$, Sau3AI, 0) and the molecular size markers (lanes MW) are indicated in base pairs (A) and kilobase pairs (B). The position of the markers relative to the partial restriction maps are indicated by lines. 


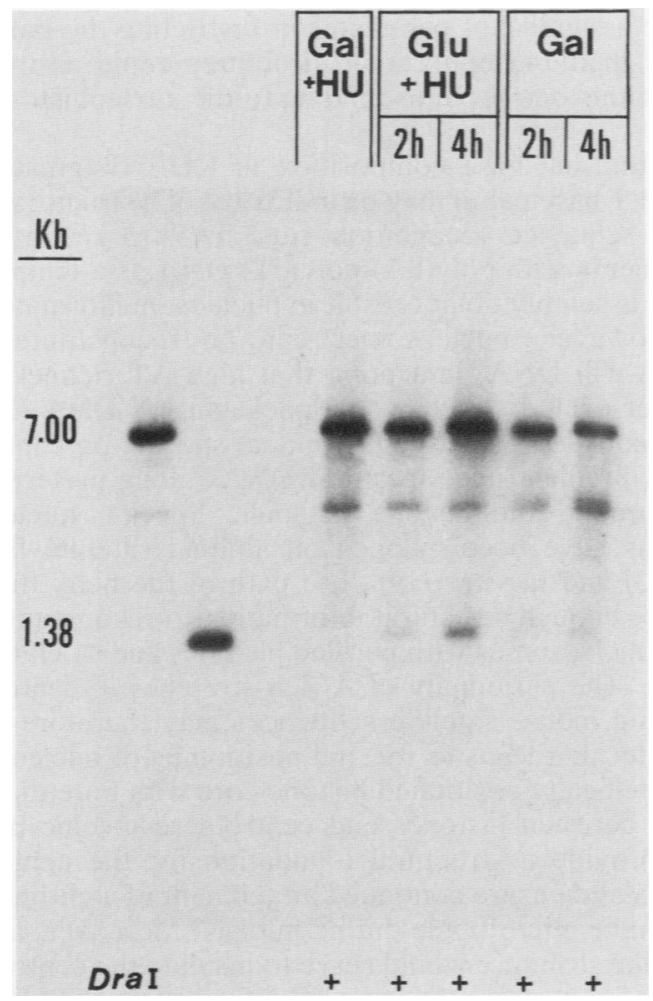

FIG. 4. Histone repression in cells arrested with HU results in centromere accessibility. UKY 403 cells were grown in galactose, and HU was added to a final concentration of $0.3 \mathrm{M}$. Cells were arrested for $5 \mathrm{~h}$ and then switched to glucose plus HU. Samples were taken at 2 and $4 \mathrm{~h}$, and cells were switched back to galactose in the absence of HU. Samples were again taken after 2 and $4 \mathrm{~h}$ more. Nuclei were isolated, and the chromatin DNA was treated for nuclease digestion with $\mathrm{DraI}(+)$, as described in the legend to Fig. 3. Samples were subsequently digested with HindIII, electrophoresed on a $1.4 \%$ agarose gel, and hybridized to a $900-\mathrm{bp}$ HindIII-Bam HI fragment to visualize the intact HindIII fragment $(7.0 \mathrm{~kb})$ and the $1.38-\mathrm{kb}$ DraI digestion product.

ceeded to the $40 \%$ level (Fig. 3 ; data not shown). When the cells were shifted to galactose in the absence of HU, DraI accessibility was diminished, as evidenced by decreased cutting $2 \mathrm{~h}$ post-induction of histone synthesis (Fig. 4, lanes, no HU). Thus the small fraction of centromeres accessible to DraI digestion in the absence of replication recovered their protected structures on histone induction. To examine whether increased accessibility of the centromere DNA in the absence of replication reflects changes in bulk chromatin DNA in cells blocked with HU, micrococcal nuclease digestion was performed on the nuclei from UKY403 cells subjected to a similar regime. Following the switch to glucose in the presence of HU (after HU pretreatment on galactose), the bulk of the chromatin exhibited nuclease cutting patterns intermediate between those of wild-type chromatin and chromatin from histone-depleted cells (data not shown). These data indicate that the significant level of accessibility to nucleases following histone depletion (Fig. 1 through 3) depended primarily on progression through the $S$ phase of the cell cycle.

The small fraction of centromeres accessible to nuclease digestion on histone repression in the absence of replication may reflect the dynamic properties of centromeric histones in particular, or as suggested above, may be a general feature of bulk nucleosomes. The ability to regulate histone levels

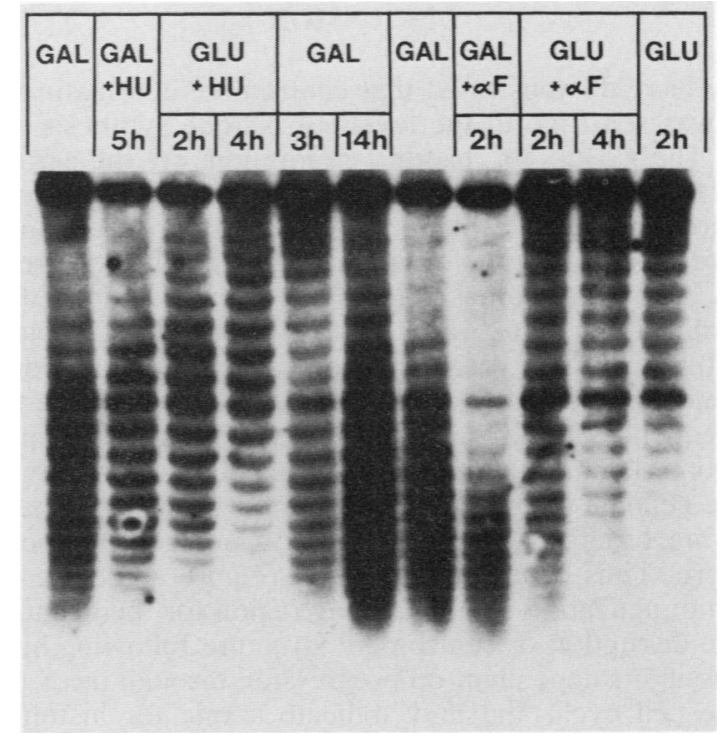

FIG. 5. Superhelicity of covalently closed circular plasmids in cells arrested with $\mathrm{HU}$ and switched from galactose to glucose. UKY 403 cells were subjected to the same growth regime described in the legend to Fig. 4. DNA was isolated and electrophoresed in a $1 \%$ agarose gel containing $1.25 \mu \mathrm{g}$ of chloroquine phosphate per ml. The DNA was transferred to nitrocellulose and hybridized to pBR322 DNA to visualize the plasmid-containing sequences. $\alpha F, \alpha$ Factor; GAL, galactose; GLU, glucose.

has provided the opportunity to quantitate changes in nucleosome distribution along the chromatin DNA in the presence or absence of replication. We measured the superhelical density of intracellular, covalently closed plasmids from cells grown in galactose and then in galactose plus $\mathrm{HU}$, depleted for histone $\mathrm{H} 4$ in glucose plus $\mathrm{HU}$, and finally returned to galactose. DNA was isolated from these cells, and plasmid DNA was electrophoresed in the presence of chloroquine phosphate to resolve individual topoisomers. On histone repression a shift to more positively supercoiled species, signifying a loss of nucleosomes, was observed for the centromere plasmid pUK421 (Fig. 5, GAL + HU versus GLU + HU lanes). Thus the distribution of nucleosomes along the chromatin DNA was altered on histone repression independent of the action of replication. $\alpha$ Factor was also used to arrest cells at the $G_{1}$ stage of the cell cycle prior to DNA replication. Inhibition of histone $\mathrm{H} 4$ synthesis in cells arrested at $G_{1}$ also resulted in a change in the superhelical density of intracellular plasmids (Fig. 5). An identical shift in the superhelical density was observed for the endogenous $2 \mu \mathrm{m}$ plasmid following both cell cycle arrests (data not shown). These data indicate that the predominant effect of histone repression on chromatin structure occurs from the inability to assemble nucleosomal particles following DNA replication. The minor changes observed in DraI accessibility of CDE II and the density shifts in plasmid topoisomers may result from a small percentage of cells that are insensitive to $\mathrm{HU}$ or $\alpha$ factor treatments. Alternatively, a dynamic equilibrium between histones and DNA may exist in the absence of DNA replication and transcription. Jackson (15) has demonstrated that nucleosomal subunits are free to exchange with histones pools without restriction to active replication and transcriptional events. A constitutive, albeit low, level of exchange between histone subunits and DNA could result in exposed regions of DNA when histone synthesis is repressed. 


\section{DISCUSSION}

We have demonstrated that centromere chromatin structure was sensitive to the levels of histone synthesis in the cell. The effects of histone repression on the structure reflects either the presence of histones at the centromere core or a role for flanking nucleosomal chromatin in centromere assembly and/or maintenance. Yeast centromere DNA can be subcloned from its endogenous sequences and introduced into host cells in a variety of sequence or structural contexts. Yeast centromeric DNA is functional in plasmids that lack any of the endogenous centromere flanking sequences (26). In addition, the presence of a specific centromeric chromatin structure embedded in other regions of the genome or in procaryotic vector sequences does not generate ordered nucleosomal arrays adjacent to the centromere. Thus centromeres do not require a specific chromatin organization in the flanking region for their function.

The disruption of centromere structure following histone repression is dependent on progression through the $S$ phase of the cell cycle and may indicate a role for histones or flanking chromatin in the assembly of centromere structure. The binding of specific centromere DNA-binding proteins may require a histone template for assembly or the presence of flanking nucleosomal chromatin. These same structures may also contribute to stability of the centromere complex during the cell cycle. Yeast centromeres severed from the flanking chromatin arrays by in situ restriction digestion continue to display their unique structural organization (17). Thus the maintenance of centromeric chromatin structure is not dependent on topological constraints within the chromosome or the presence of flanking nucleosomal arrays. A direct test of histones in centromere assembly will require an in vitro chromatin assembly system and specific centromere DNA-binding proteins.

Centromere chromatin structure is maintained throughout the yeast cell cycle as well. Yeast cells can be synchronized at $G_{1}$ by mating pheromone ( $\alpha$ factor). No changes in centromere structure have been evident at any time in the cell cycle from $\alpha$ factor synchronized cells or in a variety of cell cycle mutants ( $c d c 4, c d c 15$, and $c d c 16$ mutants), nuclear division cycle mutants ( $n d c l$ mutant), and topoisomerase mutants (topl, top2, and top3 mutants) or in stationaryphase cells (3; Bloom laboratory, unpublished data). Thus the alterations associated with centromeric chromatin following histone repression are not likely to simply reflect cell cycle alterations.

Consistent with the notion of centromeric histones is the dissociation pattern of centromeric proteins following salt elution of yeast chromatin (1). Concentrations of $\mathrm{NaCl}$ below that required for dissociation of histone proteins have no effect on the centromere core, whereas at concentrations that dissociate histones $(0.75$ to $1.25 \mathrm{M})$, there is a concomitant disruption of the centromere core. Previous nuclease studies on centromere mutations with insertions and deletions in CDE II have revealed alterations in the dimensions of the nuclease-resistant core that parallel the change in length of the centromere DNA (26). However, none of the CEN3 mutations examined exhibited a nuclease-resistant structure less than $145 \mathrm{bp}$, the size of the nucleosome core DNA. The chromatin structures of four unique centromeres, $C E N 3, C E N 4, C E N 11(1,2)$, and $C E N 14(7)$ have now been mapped, and all range from 150 to $220 \mathrm{bp}$ of chromatin DNA. Analysis of numerous mutated and wild-type centromeres has revealed protected structures that vary from 145 to 300 bp. The presence of a nucleosomal core at the centromere may be a significant parameter in restricting the particle to no less than $145 \mathrm{bp}$. Larger structures could easily result from nonhistone proteins binding to the nucleohistone complex.

The unusual base composition of CDE II (greater than $90 \% \mathrm{~A}+\mathrm{T}$ base pairs) may be indicative of a structural rather than a sequence recognition site. In vitro reconstitution experiments with poly(dA)-poly(dT) DNA as a template for histone assembly do not result in nucleosome formation (24, 28). However, nucleosomes can be reconstituted onto poly(dA-dT) DNA, indicating that high AT richness alone does not exclude nucleosome packaging of DNA $(24,28)$. Nucleosomal DNA exhibits kinks at specific positions (25), thereby predisposing structural DNAs along preferred registers around the histone octamer. Specific nucleosome positions have been mapped on alphoid satellite DNA in vivo (23) and in vitro (19). The path of the helix therefore provides enough structural information to reconstitute positioned nucleosomes with purified histones and satellite DNA in vitro. The periodicity of A-rich stretches in centromeric DNA and mouse satellite sequences may therefore be one parameter that leads to specific positioning of nucleosomes.

A specifically positioned histone core with potential interactions between histones and centromere-specific proteins could provide a structural foundation for the centromere complex and ensure continued attachment of a chromosome to a spindle microtubule during mitosis. Moreover, a nucleosomelike structure would serve to insulate the centromeres from torsional stress. Specific roles for histones in transcriptional control have been proposed recently (16). An N-terminal $\mathrm{H} 4$ deletion protein results in derepression of the silent mating type loci HML and HMR. Grunstein and co-workers have suggested that a SIR protein complex might interact with the $\mathrm{N}$ terminus of $\mathrm{H} 4$ in one or more nucleosomes located at the silencer sites (16). Involvement of a histone octamer as the centromere foundation with stabilizing contacts to centromere specific proteins may also represent a novel function for yeast histones.

\section{ACKNOWLEDGMENTS}

This work was supported by a grant from the National Institutes of Health to K.B. K.B. was supported by a Research Career Development Award from the National Cancer Institute. M.S. was awarded a Pogue fellowship from the University of North Carolina.

\section{LITERATURE CITED}

1. Bloom, K. S., E. Amaya, J. Carbon, L. Clarke, A. Hill, and E. Yeh. 1984. Chromatin conformation of a yeast centromere. J. Cell Biol. 99:1559-1568.

2. Bloom, K. S., and J. Carbon. 1982. Yeast centromere DNA is in a unique and highly ordered structure in chromosomes and small circular minichromosomes. Cell 29:305-317.

3. Bloom, K. S., M. Kenna, and M. Saunders. 1989. Cis- and Trans-acting factors affecting the structure of yeast centromeres. J. Cell Sci. Suppl. 12:231-242.

4. Clarke, L., and J. Carbon. 1980. Isolation of a yeast centromere and construction of a functional small circular chromosome. Nature (London) 287:504-509.

5. Cumberledge, S., and J. Carbon. 1987. Mutational analysis of meiotic and mitotic CEN function in Saccharomyces cerevisiae. Genetics 117:203-212.

6. Fitzgerald-Hayes, M., L. Clarke, and J. Carbon. 1982. Nucleotide sequence comparison and functional analysis of yeast centromere DNA's. Cell 29:235-244.

7. Funk, M., J. H. Hegemann, and P. Philippsen. 1989. Chromatin digestion with restriction endonucleases reveals $150-160 \mathrm{bp}$ of protected DNA in the centromere of chromosome XIV in Saccharomyces cerevisiae. Mol. Gen. Genet. 219:153-160. 
8. Gaudet, A., and M. Fitzgerald-Hayes. 1987. Alterations in the adenine-plus-thymidine-rich region of $C E N 3$ affect centromere function in Saccharomyces cerevisiae. Mol. Cell. Biol. 7:68-75.

9. Han, M., M. Chang, U. Kim, and M. Grunstein. 1987. Histone H2B repression causes cell-cycle-specific arrest in yeast: effects on chromosomal segregation, replication, and transcription. Cell 48:589-597.

10. Han, M., U. Kim, P. Kayne, and M. Grunstein. 1988. Depletion of histone $\mathrm{H} 4$ and nucleosomes activates the PHO5 gene in Saccharomyces cerevisiae. EMBO J. 7:2221-2228.

11. Hays, T. S., and E. D. Salmon. 1990. Poleward force at the kinetochore in metaphase depends on the number of kinetochore microtubules. J. Cell Biol. 110:391-404.

12. Hegemann, J. H., J. Shero, G. Cottarel, P. Philippsen, and P. Hieter. 1988. Mutational analysis of centromere DNA from chromosome VI of Saccharomyces cerevisiae. Mol. Cell. Biol. 8:2523-2535.

13. Hieter, P., D. Pridmore, J. H. Hegemann, M. Thomas, and R. W. Davis. 1985. Functional selection and analysis of yeast centromeric DNA. Cell 42:913-921.

14. Holm, C., T. Stearns, and D. Botstein. 1989. DNA topoisomerase II must act at mitosis to prevent nondisjunction and chromosome breakage. Mol. Cell. Biol. 9:159-168.

15. Jackson, V. 1990. In vivo studies on the dynamics of histoneDNA interaction: evidence for nucleosome dissolution during replication and transcription and a low level of dissolution independent of both. Biochemistry 29:719-731.

16. Kayne, P. S., U.-J. Kim, M. Han, J. R. Mullen, F. Yoshizaki, and M. Grunstein. 1988. Extremely conserved histone $\mathrm{H} 4$ terminus is dispensable for growth but essential for repressing the silent mating type loci in yeast. Cell 55:27-39.

17. Kenna, M., E. Amaya, and K. Bloom. 1988. Selective excision of the centromere chromatin complex from Saccharomyces cerevisiae. J. Cell Biol. 107:9-15.

18. Kim, U., M. Han, P. Kayne, and M. Grunstein. 1988. Effects of histone $\mathrm{H} 4$ depletion on the cell cycle and transcription of
Saccharomyces cerevisiae. EMBO J. 7:2211-2219.

19. Linxweiler, W., and W. Horz. 1985. Reconstitution experiments show that sequence-specific histone-DNA interations are the basis for nucleosome phasing on mouse satellite DNA. Cell 42:281-290.

20. McGrew, J., B. Diehl, and M. Fitzgerald-Hayes. 1986. Single base-pair mutations in centromere element III cause aberrant chromosome segregation in Saccharomyces cerevisiae. Mol. Cell. Biol. 6:530-538.

21. McNiell, P. A., and M. W. Berns. 1981. Chromosome behavior after laser microirradiation of a single kinetochore in mitotic PtK $_{2}$ cells. J. Cell Biol. 88:543-553.

22. Meeks-Wagner, D., and L. H. Hartwell. 1986. Normal stoichiometry of histone dimer sets is necessary for high fidelity of mitotic chromosome transmission. Cell 44:43-52.

23. Musich, P. R., F. Brown, and J. J. Maio. 1982. Nucleosome phasing and micrococcal nuclease cleavage of African green monkey component $\alpha$-DNA. Proc. Natl. Acad. Sci. USA 79: 118-122.

24. Rhodes, D. 1979. Nucleosome cores reconstituted from poly $(\mathrm{dA} \cdot \mathrm{dT})$ and the octamer of histones. Nucleic Acid Res. 6:1805-1816.

25. Richmond, T. J., T. J. Finch, B. Rushton, D. Rhodes, and A. Klug. 1984. Structure of the nucleosome core particle at 7A resolution. Nature (London) 311:532-537.

26. Saunders, M., M. Fitzgerald-Hayes, and K. Bloom. 1988. Chromatin structure of altered yeast centromeres. Proc. Natl. Acad. Sci. USA 85:175-179.

27. Shure, M., D. F. Pulleybank, and J. Vinograd. 1977. The problems of eukaryotic and prokaryotic DNA packaging and in vivo conformation posed by superhelix density heterogeneity. Nucleic Acids Res. 4:1183-1205.

28. Simpson, R. T., and P. Kunzler. 1979. Chromatin and core particles formed from the inner histones and synthetic polynucleotides of defined sequence. Nucleic Acid Res. 6:1387-1415. 